\title{
Offer and veto: an experimental comparison of two negotiation procedures
}

\author{
Michael Filzmoser $^{1}$ (D) . Johannes R. Gettinger ${ }^{1}$
}

Received: 1 January 2018 / Accepted: 6 December 2018 / Published online: 15 December 2018

(c) The Author(s) 2018

\begin{abstract}
Negotiation by veto is introduced as a novel negotiation approach and as an alternative to the exchange of offers. Rather than proposing offers, negotiators following the negotiation by veto approach eliminate unfavorable settlement options from the set of possible agreements until they eventually achieve a mutual acceptable solution. It is argued that this approach could lead to superior negotiation outcomes and improve negotiators' satisfaction. In an experiment with student participants the performance of offer and veto negotiation procedures is compared. In simple negotiation problems both negotiation procedures reach similar outcomes. In complex negotiation problems negotiation by veto achieves fewer but better agreements. However, participants were more satisfied with the negotiation process, outcome and their opponent's behavior when exchanging offers rather than vetoing alternatives.
\end{abstract}

Keywords Negotiation · Process · Offer exchange $\cdot$ Veto

Mathematics Subject Classification 91A35 - 91 A90 · 91B06 · 91B26

\section{Introduction}

Negotiation is a coordination mechanism that can be found in many domains of life. Salary negotiations, sales and procurement negotiations, collective bargaining and labor disputes, law and court, to give only a few examples. Negotiations are applied to settle conflicts or align differing interests between individuals, companies or whole nations. In general negotiation is a joint decision making process with the aim to transform initially conflicting interests into a situation mutually acceptable for all negotiators Zartman (1977). Negotiation, therefore, is a mixed-motive task as the motive, to maximize individual outcomes, and the motive, to collectively reach a beneficial agreement, have to be orchestrated Bartos (1977), Dupont and Faure (2002), Lewicki et al. (1994).

Michael Filzmoser

michael.filzmoser@tuwien.ac.at

1 Institute of Management Science, TU Wien, Vienna, Austria 


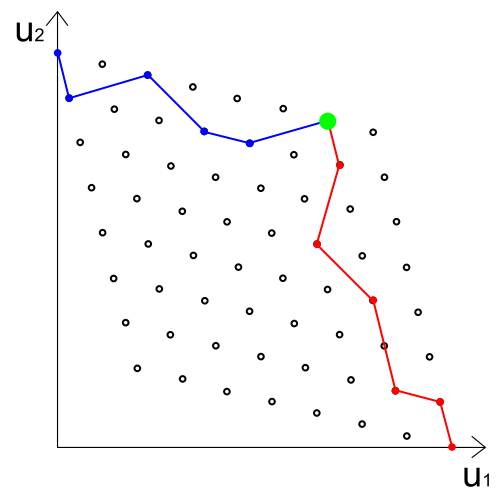

(a) Concession-based progress

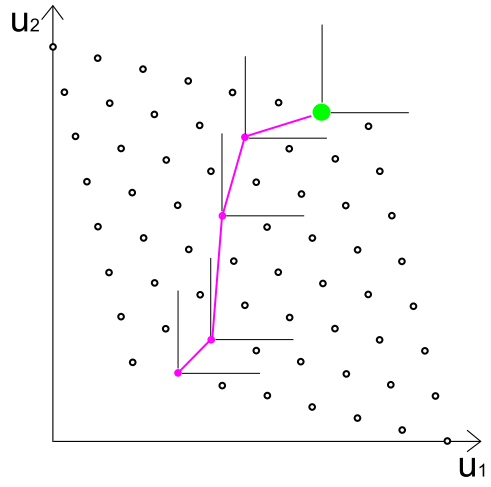

(b) Improvement-based progress

Fig. 1 Concession-based and improvement-based progress in negotiations

Negotiations are typically accomplished by exchanging offers, as in most cases the parties do not settle on the first offer on the bargaining table Mumpower (1991). Therefore, the exchange of offers is an important form of communication in negotiations. Management science or economics often model negotiations as offer exchange process Tutzauer (1992). Though commonly used, exchanging offers could also impede reaching a satisfactory agreement in negotiations, by unfortunate steps or if these offers are perceived an defended as positions in positional bargaining procedures Fisher and Ury (1981). Negotiators that engage in so-called negotiation dances, after their opening offers step-wise reduce their claims by concessions to proceed incrementally and cautiously to an agreement in the hope not to be exploited by the opponent Mumpower (1991). A concession is an attempt to make the opponent better off in suggesting a solution that features higher utility for the opponent at the cost of lower own utility Filzmoser and Vetschera (2008). However, as the preferences of the opponent typically are unknown, negotiators could mistakenly do unfortunate steps that make all parties worse off. Furthermore, proposing an offer, as a possible settlement for the negotiation, can be seen as taking a specific position in this negotiation, which could lead to positional bargaining Fisher and Ury (1981). Defending such a position is related to a number of problems in negotiations. Positional bargaining can cause inefficiently long and conflicting negotiations and harm the relationship between the negotiation parties or even impede reaching an agreement due to the inflexibility of negotiators and the feeling of losing face when giving up a position Fisher and Ury (1981).

Regardless of its potential drawbacks, concession-based offer exchange is dominant in negotiation research and practice. However, other ways of progression in negotiations exist, one of which is improvement-based progression Teich et al. (1994). Concession-based and improvement-based progression are depicted in Fig. 1. Following an improvement-based progression the negotiation parties start at a common basis, e.g. the status quo of a conflict situation or values reflecting their best alternatives to a negotiated agreement. Subsequently they try to stepwise improve this basis through a number of tentative agreements that dominate the 
previous ones until no Pareto-improvements are left or can be found, typically supported by a mediator that facilitates the procedure.

Negotiation by veto, derived from voting by veto as described in detail in the next section, is a negotiation approach based on improvement-based progression in negotiation. The approach requires negotiators to alternate in excluding undesired settlement options from the set of possible alternatives until only one alternative is left, which then can be accepted or otherwise the negotiations are terminated without an agreement. If negotiators consecutively exclude the, from their individual perspective, worst options the procedure implies a continuous improvement of the minimal utility of the set of remaining alternatives. As dominated alternatives are eliminated, in the end a desirable Pareto-efficient outcome should be obtained. The research questions of this paper are: how can the veto procedure be implemented in negotiations, as well as how do negotiation by veto perform compared to negotiation by offer exchange in terms of negotiation outcomes and satisfaction of the negotiators with the procedure? To address these research questions laboratory negotiation experiments were developed, conducted and analyzed.

The remainder of this paper is structured as follows: Sect. 2 presents the negotiation by veto approach and compares its characteristics to related approaches in decision making and negotiation literature. Furthermore, hypotheses about the effects of the offer exchange and the veto approach on negotiation outcomes and negotiators' satisfaction in complex and simple negotiation problems are developed. Section 3 presents the laboratory negotiation experiments conducted to compare the offer exchange and the veto approach. Section 4 presents the results of the experiments and the statistical analyses of differences in the treatments with respect of outcome and satisfaction measures. Section 5 summarizes the results and concludes the paper with a discussion of limitations and an outlook on future research.

\section{Negotiation by veto}

Negotiation by veto is derived from the concept of voting by veto from public choice - a research field that applies theories of group decision making, economics and game theory to research questions in political science. Voting by veto was introduced in Müller (1978) and found consideration and further developments as a voting scheme that reaches results by elimination of alternatives Moulin (1979), Yuval (2002). The approach requires, that each of $n$ voters suggest a proposal so that together with the status quo-or some other reference alternative-a total of $n+1$ alternatives exist from which the decision makers have to choose. A voting order is determined randomly and following this order each voter vetoes one alternative, i.e. eliminates this alternative from the set of possible solutions. After all voters vetoed, one alternative remains and is declared as the solution of the group decision problem.

To illustrate the procedure consider the example of three decision makers A, B and $\mathrm{C}$. The decision makers formulated, besides the status quo s, their three alternatives $\mathrm{a}, \mathrm{b}$ and $\mathrm{c}$, and their ranking of these alternatives as represented in Table 1. Let the veto order $\mathrm{B}$ followed by $\mathrm{C}$ and $\mathrm{A}$ be the result of random determination. 
Table 1 Voting by vetoillustrative example

\begin{tabular}{llll}
\hline Rank & A & B & C \\
\hline 1 & a & b & c \\
2 & c & a & s \\
3 & s & c & b \\
4 & b & s & a \\
\hline
\end{tabular}

Assuming voters veto the worst alternative from their individual perspective, B would veto $\mathrm{s}, \mathrm{C}$ would veto the alternative a and $\mathrm{A}$ would veto alternative $\mathrm{b}$ so that $\mathrm{c}$ is the solution to the group decision problem under the voting by veto decision procedure.

It is argued that voting by veto should tend to results in more balanced and agreeable solutions Müller (1978), which of course are not only desirable properties for voting outcomes but also for agreements in negotiations. Moreover, a procedure that reduces the set of possible alternatives by inferior candidates, as it is the case with a veto procedure, might be easier to accept and follow by a negotiator than a procedure that reduces the set of possible alternatives by favored candidates, as it is the case when offers made are rejected by the opponent. In negotiations, where the number of possible solutions can exceed the number of negotiators, modifications of the procedure are necessary to make the veto procedure applicable. The proposed procedure of negotiation by veto, similarly to voting by veto, starts by randomly determining the negotiator to begin the veto process. Thereafter, negotiators alternate in eliminating possible solutions until only one solution remains. Finally, the negotiators decide whether or not they agree on this remaining solution. While in voting by veto with only two voters the voter who vetoes first has a considerable advantage in case of only few alternatives-e.g. only the necessary three alternatives voting by veto requires as discussed above-in negotiation by veto this first mover advantage diminishes with the number of possible solutions in the negotiation.

For negotiation by veto to be applicable a fully specified and constant negotiation problem is required. This means that all issues of the negotiation object and all possible options for settling these issues and, therefore, all possible solutions of the negotiation problem are specified and do not change during the negotiation. Furthermore, the negotiators have to have clearly specified preferences over these possible solutions and these preferences also have to remain constant for the duration of the negotiation.

\subsection{Related literature}

Several strands in decision making and negotiation research that share similar features but are nevertheless distinct from the introduced negotiation by veto approach have to be mentioned. First of all, although quite different at the first sight, there exist overlaps with the concession-based offer exchange procedure. A rejection of an offer, as a proposed solution for the conflict, is actually nothing else than a veto as an agreement requires mutual acceptance. So negotiation by veto uses an existing 


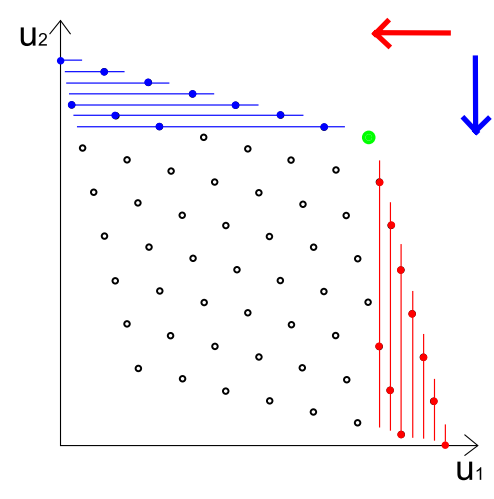

(a) Concession-based progress

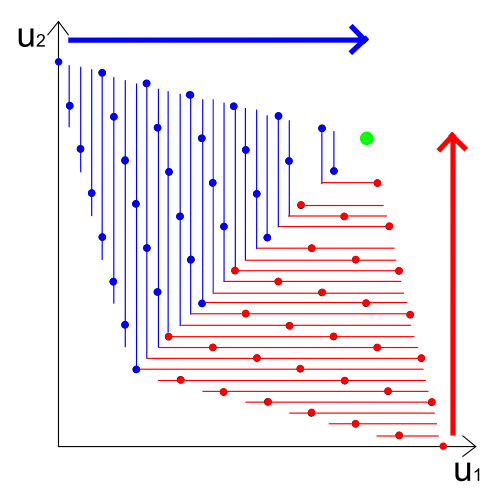

(b) Improvement-based progress

Fig. 2 Systematic concessions vs. systematic vetoes

mechanism as the basis for resembling a novel approach that focuses on systematically eliminating in advance unfavorable solutions before they have been proposed as offers.

A similarly systematic approach, also from the domain of offer exchange procedures, was proposed in Kelley (1966). The approach is called systematic concession making strategy and determines the formulation of offers during a negotiation for a negotiation party to ensure a movement towards the Pareto-frontier of the negotiation problem. Negotiators start with an extreme opening offer, if their offer is not accepted by the opponent, they alternate in proposing other offers of same utility from their perspective. The sequence in which these offers of same utility are proposed is arbitrary as the negotiator is indifferent between offers of equal utility. If all offers for a given level of utility have been proposed the negotiator lowers the demanded utility level to the next lower one and again proposes all offers of this utility level. An offer of the opponent is accepted if the utility levels demanded by the two negotiators intersect, i.e. if the next offer to propose according to the algorithm would yield equal or even lower utility compared to the current offer of the opponent. Such agreements then are likely to present a Pareto-optimal solutions as found in experimental studies Kelley (1966). Figure 2 compares the systematic concession making strategy—of always offering the best alternative in the remaining set of possible alternatives-with the negotiation by veto introduced in the previous section and assuming that consecutively the worst alternatives are eliminated from the remaining set of possible alternatives. Note that in Fig. 2 the arrows indicate the path of the negotiators by systematic offersin Fig. $2 a-$ and systematic vetoes-in Fig. $2 b$ to an agreement in the negotiation-as no such systematic offers or vetoes occur in the negotiation procedures illustrated in Fig. 1 arrows are omitted there. From Fig. 2 can be derived that consecutively offering the possible solution with highest or consecutively vetoing the possible solution with lowest utility from the set of remaining solutions always leads to agreements and always leads to equal results for the two approaches in symmetric negotiation problems, only the duration-measured in the offer or veto 
steps necessary to reach the outcome-varies between the two approaches. Which agreement can be reached following these systematic procedures depends on the shape of the negotiation problem. The equivalence-with respect to outcomesof systematic offer exchange and systematic vetoing for symmetric negotiation problems is supported by the findings of computational experimentation using the negotiation problems introduced in Mumpower (1991) and time-based and problem-based concession strategies as well as an improvement-based vetoing strategy Filzmoser (2018).

There exists some work in the multi-criteria decision making literature that uses similar approaches as negotiation by veto to decision making problems Abonyi (1983). However, the focus there is on collective choice rather than negotiation so a more collaborative situation can be assumed. Moreover, not single alternatives are eliminated but an aspiration level is defined and alternatives are evaluated by filtering them in the two set, one of alternatives which fulfill and one with alternatives that violate the aspiration level.

The improvement-based progression in negotiation mentioned in the introduction, which is also the basis for negotiation by veto, is closely related to the 'single negotiation text' (SNT) procedure developed in Fisher (1978). Following this procedure negotiators, typically supported by a mediator, first have to determine a common basis and use this as a reference point from which to move to other solutions, which are preferred over the current tentative solution by at least one participant-i.e. a Pareto-improvement. Pareto-improvement steps are repeated until no further improvements are possible or can be found. In contrast to the negotiation by veto approach developed in the previous section SNT requires joint movementstypically supported by a mediator-towards Pareto-superior alternatives, rather than improving the possible outcomes of a negotiation by eliminating undesired alternatives from the set of possible outcomes-which can be done unilaterally and, therefore, does not require the support of a third party. SNT enables a series of tentative agreements and the sense of joint progress and improvement on the way to a final solution in the negotiation, however, applying SNT and reaching intermediate consent might be difficult in a very conflicting or emotionally-laden context.

While it is impossible to use both forms of progress-concession-based and improvement-based progress-simultaneously in a negotiation they can be used sequentially. One form of such a combination is the joint search for Pareto-superior results after an initial tentative agreement was reached-often as a result of concession-based negotiation approaches. A post-settlement phase Raiffa (1985), Gettinger et al. (2016) provides the opportunity to engage in value creating steps and thereby overcome the often observed Pareto-inefficiency of outcomes. Pareto-improvements, i.e. superior outcomes for at least one party with no drawbacks for the remaining ones, are possible whenever the negotiators do not reach the Pareto-optimal frontier of their negotiation problem with a first tentative agreement. For complex negotiations, scholars argue that the negotiation parties should always consider the option of a post-settlement phase or even implement it as a working paradigm Bazerman et al. (1987). Pareto-improvement functions are common features of negotiation 
support systems that provide support also for the post-settlement phase Kersten and Noronha (1999), Rangaswamy and Shell (1997).

\subsection{Hypotheses}

The systematic consideration of all alternatives and the elimination of, typically unfavorable, alternatives from the set of possible solutions should enable negotiations using the veto procedure to achieve better outcomes than those reached with offer exchange. This should be at least the case for negotiations with a high number of potential solutions-i.e. more complex negotiation problems. In such negotiations the systematic consideration of all alternatives bears the largest benefits, e.g. when negotiators consecutively eliminate the worst of the remaining possible solutions from their perspective. In less complex negotiation problems the advantage should not be that significant. Concerning the negotiation outcome, therefore, the following hypotheses are formulated:

H1a In simple negotiation problems negotiation by veto leads to the same outcomes as negotiation by offer exchange.

H1b In complex negotiation negotiation by veto leads to better outcomes than negotiation by offer exchange.

Furthermore, eliminating unfavorable solutions by oneself could also be easier to accept than the rejection of favored offers by the opponent, as it is the case in negotiations based on offer exchange. Unaccepted and rejected offers imply that further concessions are necessary to reach an agreement and the remaining settlement options are of lower value, as the prospected maximum outcome decreases with each concession step. On the other hand with the veto approach unfavorable alternatives are eliminated from the set of possible alternatives so that the remaining solutions have higher utility and the prospected minimal utility of a final agreement increases with each veto step. Negotiation by veto, therefore, should positively influence the satisfaction of the negotiators' with the negotiation and related aspects. However, in complex negotiation problems the systematic consideration of all settlement alternatives in negotiation by veto might take longer time and cause more effort than negotiation based on offer exchange, where larger concession steps could speed up the negotiation process. These drawbacks could outweigh the satisfaction benefits especially in complex negotiations with many possible alternatives compared to simple negotiation problems. Therefore, with respect to negotiators' satisfaction the following hypotheses are formulated:

H2a In simple negotiation problems negotiation by veto leads to higher satisfaction of the negotiators than negotiation by offer exchange.

$\mathrm{H} 2 \mathrm{~b}$ In complex negotiation problems negotiation by veto leads to similar satisfaction of the negotiators than negotiation by offer exchange. 


\section{Methods}

To test the hypotheses formulated in the previous section a laboratory experiment was designed and conducted. The negotiation case is a bilateral negotiation about a specific second-hand laptop between students. The two open issues are the price of the laptop and the delivery time as shown in Table 3.

The complexity of the negotiation problem was manipulated by implementing two treatments with negotiation problems with different numbers of possible solutions within the same range of minimum and maximum values and the same range of utility scores - from 0 to 100 points. The simple treatment consists of 25 and the complex treatment of 100 possible solutions resulting from the Cartesian product of the five and ten settlement options in the two issues price and delivery time. The negotiation problem structure was designed to feature integrative potential by assigning different weights to the issues for the two parties as well as by determining concave partial utility functions Mumpower (1991). To manipulate complexity by the number of settlement options - five versus ten in the two issues which results in 25 or 100 possible solutions in the simple and complex treatment (see Table 2) was chosen to keep the shape of the negotiation problem identical and make the results in the treatments comparable. The simple and complex negotiation problems used in the experiments for this study are illustrated in Fig. 3.

In the negotiation by veto treatment, the negotiators alternated in eliminating possible solutions until only one possible solution remained. The parties were not restricted to eliminate only the worst solution from their perspective but could eliminate any of the remaining solutions from the set of possible solutions. Finally, the negotiators decided whether or not they accept the last remaining alternative as agreement of their negotiation. In the negotiation treatment with offer exchange the negotiators alternated in making offers and the negotiation ends if one negotiator accepts the offer of the opponent, no further restrictions were imposed in this treatment. Participants did not have to consider all possible solutions, as in the negotiation by veto treatment, but could accept any offer made by their opponent. The two negotiation procedures (offer and veto) and the two negotiation problem (simple and complex) combine to a $2 \times 2$ experimental design as illustrated in Table 3 .

In all treatments the same procedure was applied to gather data. Participants received a general introduction about the second-hand laptop negotiation in the form of public information, which was identical for both parties. In addition participants received private information about the preferences over the settlement options only for the role they assumed in the negotiation experiment and a scoring table listing all possible solutions and the related utility values from the perspective of their role. The participants determined which party starts the alternating negotiation procedure

Table 2 Negotiation object in the simple and complex treatments

\begin{tabular}{lll}
\hline Issues & Simple & Complex \\
\hline Price $(350-490 €)$ & 5 & 10 \\
Delivery $(1-10$ days $)$ & 5 & 10 \\
Number of possible solutions & $5 \times 5=25$ & $10 \times 10=100$ \\
\hline
\end{tabular}



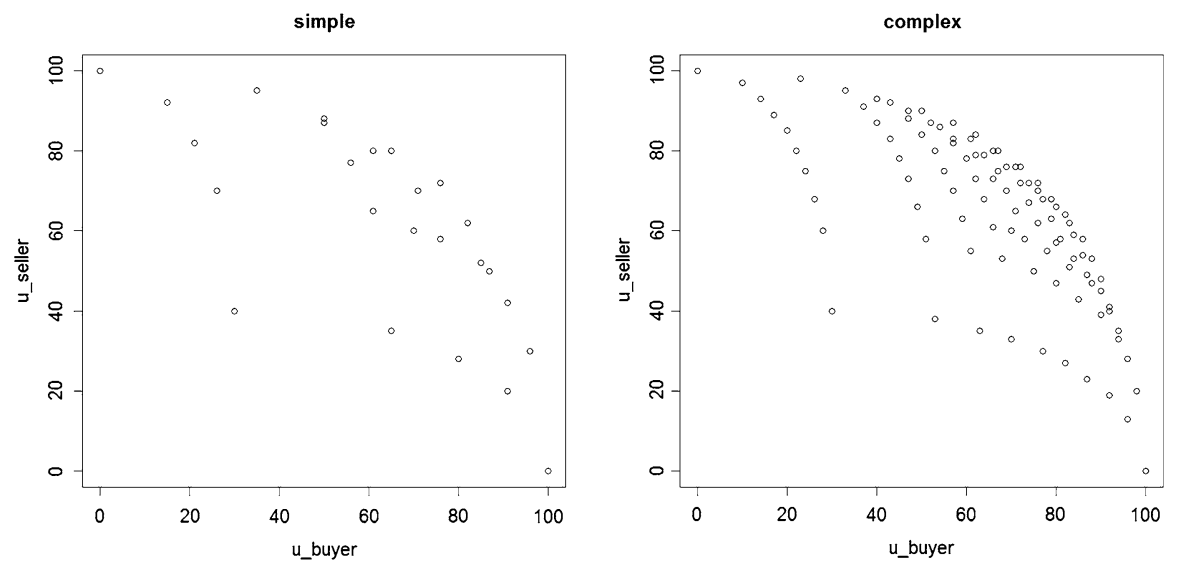

Fig. 3 Simple and complex negotiation problem

Table 3 Experimental design

\begin{tabular}{cll}
\hline & Complexity & \\
\cline { 2 - 3 } & Low & High \\
\hline $\begin{array}{c}\text { Procedure } \\
\text { Offer }\end{array}$ & Offer-simple & Offer-complex \\
Veto & Veto-simple & Veto-complex \\
\hline
\end{tabular}

randomly, by tossing a coin. In the treatments with simple negotiation problems the available negotiation time was restricted to $15 \mathrm{~min}$ in the treatments with complex negotiation problems $30 \mathrm{~min}$ were available to conduct the negotiation. These durations were found to be sufficient to conduct the negotiations with the simple and complex negotiation problem in pre-tests. The private information of the parties included a list of all 25 , respectively, 100 possible solutions and their utility values to the focal party. During the negotiation the participants had to record the order of offers or vetoes, depending on the treatment, in an empty column in this table of possible alternatives. After the negotiations were finished the participants answered a questionnaire providing demographic information and subjective evaluations of their satisfaction with the negotiation process, the achieved outcome, their opponent and the negotiation climate. Multi-item constructs and questions from previous negotiation research, reflecting post-negotiation satisfaction, were used for this purpose, the questionnaire can be obtained from the authors upon request. Each of the four constructs was measured by four items on a seven point Likert-scale. 
Table 4 Number of observations (dyads) in the experimental treatments

\begin{tabular}{lc}
\hline Treatment & Dyads \\
\hline Offer-simple & 23 \\
Veto-simple & 33 \\
Offer-complex & 33 \\
Veto-complex & 21 \\
Total & 110 \\
\hline
\end{tabular}

\section{Results}

Data were collected over a period of two years, during which face-to-face laboratory experiments with student participants of negotiation and management courses at the Vienna University of Technology and University of Vienna were conducted. A total of 292 students participated in 146 negotiation dyads in the experiments. Students were assigned randomly to dyads and roles. $36 \%$ of the participants were female and the average age of the participants was 25.1 years. Students received course credits for their participation independent of the outcome they achieved in the negotiation experiment. Participation-based rather than performance-based remuneration was chosen for the study as pre-tests indicated high intrinsic motivation of the participants to perform well and as it would be inappropriate to base remuneration on performance in randomly assigned treatments that impose negotiation procedures hypothesized to positively and negatively influence the negotiation outcomes in those treatments. From the full record of negotiations 18 dyads were excluded due to missing questionnaire answers, and another 18 dyads as they violated the veto procedure described above. Those dyads that deviated from the experiment instructions in the negotiation by veto treatment did so in agreeing after several vetoes on a solution ahead of schedule before all possible solutions were vetoed and only one remained as potential outcome. Table 4 reports the remaining observations in the four experimental treatments, differences in the treatment sizes result, besides exclusions, from different course sizes.

\subsection{Negotiation outcomes}

Negotiation outcomes are operationalized by several measures to cover different aspects of the negotiation's effectiveness as well as the quality of the outcome, i.e. efficiency and fairness. Existence and efficiency of an agreement in a negotiation are often found to be two conflicting goals. Aspects in a negotiation that support reaching an agreement, such as low opening offers, many and large concessions, often simultaneously lead to an agreement of lower quality, i.e. an inefficient agreement or an agreement farther away from the Pareto-frontier. This phenomenon is often called the negotiation dilemma Pruitt (1981), Filzmoser (2010a). The agreement rate, i.e. the percentage of negotiations that ended with an agreement in each treatment Tripp and Sondak (1992), was used as measure for the negotiation procedure's effectiveness. Concerning the quality of reached agreements, i.e. efficiency and fairness, 
Table 5 Agreement and Pareto-efficient agreement rates

\begin{tabular}{llll}
\hline & Agreement rate & $\chi^{2}(d f=1)$ & $p$ value (2-tailed) \\
\hline Offer-simple & $23(100 \%)$ & & 0.071 \\
Ceto-simple & $28(85 \%)$ & 3.827 & \\
Offer-complex & $33(100 \%)$ & & 0.006 \\
Veto-complex & $16(76 \%)$ & 8.659 & $p$ value (2-tailed) \\
\hline & Pareto-optimal & $\chi^{2}(d f=1)$ & 1.000 \\
\hline Offer-simple & $17(73 \%)$ & & \\
Veto-simple & $21(75 \%)$ & 0.008 & \\
Offer-complex & $2(6 \%)$ & & \\
Veto-complex & $3(19 \%)$ & & \\
\hline
\end{tabular}

several other outcome measures were considered. The rate of Pareto-efficient agreements was calculated for those negotiations in the treatments that reached an agreement. Furthermore, the minimal Euclidean distance to the Pareto-frontier was calculated to investigate how far reached solutions are from the Pareto-optimal frontier. On the other hand the contract imbalance, i.e. the absolute difference between the utility of the two parties, was calculated as a measure of fairness. Of course a high agreement rate and a high rate of Pareto-optimal agreements indicate good negotiation outcomes while for a good negotiation outcome the minimal Euclidean distance to the Pareto-frontier and for a fair one the contract imbalance should be low.

Table 5 presents the agreement rates and rates of Pareto-optimal agreements (for negotiations that reached an agreement) in the four treatments. $\chi^{2}$ tests of independence indicate, that in the offer procedure the high integrative potential of, both the simple and the complex, negotiation problem was exploited by the participants. However, the agreement rate with the negotiation by veto approach was significantly lower when negotiating complex problems, and weakly significant $(p=0.071)$ for simple negotiation problems. The shares of Pareto-optimal agreements in the simple negotiation problem differ only insignificantly between the offer and the veto procedure. For the complex negotiation problem $\chi^{2}$ tests could not be applied to test the differences in shares of Pareto-optimal agreements due to the low number of observations (less than five) in some cells, but are quite similar.

The results of comparisons of the minimal Euclidean distance to the Pareto-frontier and the contract imbalance are presented in Table 6. Shapiro-Wilks tests of normality indicate deviation from normal distribution of these outcome measures so non-parametric Wilcoxon rank sum tests were applied to test differences in these two outcome measures between the treatments.

Analyses show no effect of the negotiation procedure in negotiation problems with low complexity but in complex negotiation problems the participants applying negotiation by veto achieved results significantly closer to the Pareto-frontier. The solutions were also more balanced and, therefore, fairer in the negotiation by veto treatments, which was, however, only weakly significant $(p=0.069)$ for the simple negotiation problem as can be derived from Table 6 . 
Table 6 Distance to the Paretofrontier and contract imbalance

Table 7 Principal factor analysis for satisfaction constructs

\begin{tabular}{llll}
\hline & Mean & $W$ & $p$ value (2-tailed) \\
\hline Distance to Pareto-frontier & & \\
Offer-simple & 1.45 & & \\
Veto-simple & 1.64 & 319.5 & 0.947 \\
Offer-complex & 3.15 & & \\
Veto-complex & 2.39 & 184.5 & 0.042 \\
Contract imbalance & & & \\
Offer-simple & 15.17 & & \\
Veto-simple & 8.04 & 230.0 & 0.069 \\
Offer-complex & 12.15 & & \\
Veto-complex & 7.44 & 166.5 & 0.036 \\
\hline
\end{tabular}

\begin{tabular}{lllll}
\hline & Process & Outcome & Opponent & Climate \\
\hline PRO1 & 0.653 & & & \\
PRO2 & 0.477 & & & \\
PRO3 & 0.535 & & & \\
OUT1 & & 0.753 & & \\
OUT2 & & 0.970 & & \\
OUT3 & & 0.868 & & \\
OUT4 & & 0.822 & & \\
CP1 & & & 0.832 & \\
CP2 & & & 0.691 & \\
CP3 & & & 0.905 & \\
CP4 & & & 0.820 & \\
CL1 & & & & 0.647 \\
CL2 & & & & 0.878 \\
CL3 & & & & 0.841 \\
Cronbach's alpha & 0.727 & 0.937 & 0.916 & 0.878 \\
\hline
\end{tabular}

\subsection{Post-negotiation satisfaction}

For the multi-item measurement of the four satisfaction constructs of the post-negotiation questionnaire a principal factor analysis was conducted to test the reliability and validity. An overview of the results is provided in Table 7. Two items were eliminated due to lack of factor loading otherwise the items loaded as intended. The principal factor analysis explained $72.55 \%$ of the variance and the Cronbach's alpha values for all four constructs are above 0.7 , which is satisfactory for exploratory studies.

The factor scores derived from the principal factor analysis are used in the subsequent analyses. These factor scores for the four satisfaction constructs for the two negotiation parties were added to achieve dyad level satisfaction measures. 
Table 8 Self-reported satisfaction measures

\begin{tabular}{|c|c|c|c|}
\hline & $\begin{array}{l}\text { Satisfaction meas- } \\
\text { ure (factor scores) }\end{array}$ & $r$ & $p$ value (2-tailed) \\
\hline \multicolumn{4}{|c|}{ Process $(F(3,106)=7.848, p<0.001)$} \\
\hline Offer-simple & 0.148 & & \\
\hline Veto-simple & -0.309 & 0.261 & 0.112 \\
\hline Offer-complex & 0.477 & & \\
\hline Veto-complex & -0.427 & 0.500 & $<0.001$ \\
\hline \multicolumn{4}{|c|}{ Outcome $(F(3,106)=3.636, p=0.015)$} \\
\hline Offer-simple & 0.230 & & \\
\hline Veto-simple & -0.271 & 0.249 & 0.251 \\
\hline Offer-complex & 0.339 & & \\
\hline Veto-complex & -0.338 & 0.364 & 0.073 \\
\hline \multicolumn{4}{|c|}{ Opponent $(F(3,106)=5.661, p<0.001)$} \\
\hline Offer-simple & 0.228 & & \\
\hline Veto-simple & -0.410 & 0.299 & 0.066 \\
\hline Offer-complex & 0.419 & & \\
\hline Veto-complex & -0.264 & 0.386 & 0.049 \\
\hline \multicolumn{4}{|c|}{ Climate $(F(3,106)=0.410, p=0.746)$} \\
\hline Offer-simple & -0.118 & & \\
\hline Veto-simple & -0.071 & 0.023 & 1.000 \\
\hline Offer-complex & 0.137 & & \\
\hline Veto-complex & 0.024 & 0.061 & 1.000 \\
\hline
\end{tabular}

As the resulting dyad level satisfaction measures only slightly deviate from normal distribution factorial analysis of variance can be applied to investigate differences between treatments. The effect sizes and significance values of the factorial analysis of variance and the related post-hoc tests for the comparisons of the differences between offer and veto procedure in simple and complex negotiation problems for all four satisfaction measures are summarized in Table 8 .

Concerning the post-negotiation satisfaction with the negotiation process we observe higher satisfaction with the offer exchange procedure than with the veto procedure in both the simple and the complex treatment, however, this difference is only significant for complex negotiation problems. A similar result is obtained for the satisfaction with the outcome of the negotiations. Also for the outcome, satisfaction was higher when it was achieved using the offer exchange rather than the veto approach and again this difference was weakly significant $(p=0.073)$ only for complex negotiation problems. This result corresponds to prior research that indicates that satisfaction with negotiation process and outcome are interrelated Curhan et al. (2006). Concerning the satisfaction with the opponent and its behavior we find no differences for complexity, the reported satisfaction is significantly higher for both the simple negotiation problems $(p=0.066)$ and even more so for complex negotiation problems $(p=0.049)$. Finally, the satisfaction 
Table 9 Overview of the results for negotiation outcome

\begin{tabular}{lll}
\hline H1 outcome & $\begin{array}{l}\text { H1a } \\
\text { (Veto-simple = offer-simple) }\end{array}$ & $\begin{array}{l}\text { H1b } \\
\text { (Veto-complex }>\text { offer-complex) }\end{array}$ \\
\hline $\begin{array}{ll}\text { Agreement rate } \\
\text { Efficient rate }\end{array}$ & $\begin{array}{l}\text { Confirmed } \\
\text { Confirmed }\end{array}$ & $\begin{array}{l}\text { Reject (sig. opposite) } \\
\text { Too few observations }\end{array}$ \\
\hline H1 outcome & H1a & H1b \\
& $($ Veto-simple $=$ offer-simple) & (Veto-complex $<$ offer-complex) \\
\hline Dist. to eff. frontier & Confirmed & Confirmed \\
Contract imbalance & Confirmed & Confirmed \\
\hline
\end{tabular}

with the negotiation climate was evaluated slightly better in complex than in simple negotiation problems, probably due to the longer period of interaction, however, this effect was not significant and no effects of the negotiation procedure on satisfaction with the negotiation climate could be observed.

\section{Discussion and conclusion}

This paper aimed at proposing and evaluating negotiation by veto as an alternative to offer exchange in negotiations. We find that the complexity of the negotiation problem-measured by the number of possible solutions-influences the performance of the used negotiation procedure. In simple negotiation problems the performance of both negotiation procedures is not significantly different. However, in complex negotiation problems we find a typical example of the negotiation dilemma: negotiation by veto is the procedure leading to the better results but at the same time reduces the prospects of reaching an agreement. Table 9 provides an overview of the results on negotiation outcomes with respect to the hypotheses formulated for the experimental study. Hypothesis H1a can be confirmed and also $\mathrm{H} 1 \mathrm{~b}$ except for the agreement rate in complex negotiations were the vetoing procedure performs worse, which could be a result of the negotiation dilemma.

Concerning post-negotiation satisfaction we find that the participants were more satisfied with process, outcome and opponent in offer exchange negotiations than in the vetoing treatments in complex negotiation problems. This was at least weakly significant for the complex negotiation problems. We, therefore, reject hypothesis $\mathrm{H} 2 \mathrm{a}$ that negotiation by veto is more satisfying than offer exchange for simple problems also for some satisfaction measures offer exchange performed better than vetoing contrary to hypothesis $\mathrm{H} 2 \mathrm{~b}$. It seems that the drawbacks of negotiation by veto, i.e. the higher efforts due to the systematic consideration of all alternative solutions as well as the unfamiliarity with this novel negotiation approach outweigh potential benefits already for simple negotiation problems and even more so for complex ones. One possible explanation for these observations is that it could be easier for the negotiators to consider and offer few alternatives that are acceptable and an unsatisfactory endeavor to veto many unacceptable alternatives. The negotiations 
Table 10 Overview of the results for post-negotiation satisfaction

\begin{tabular}{lll}
\hline H2 satisfaction & $\begin{array}{l}\text { H2a } \\
\text { (Veto-simple }>\text { offer-simple) }\end{array}$ & $\begin{array}{l}\text { H2b } \\
\text { (Veto-complex = offer-complex) }\end{array}$ \\
\hline Process & Reject & Reject (offer sig. higher) \\
outcome & Reject & Reject (offer sig. higher) \\
Opponent & Reject (offer sig. higher) & Reject (offer sig. higher) \\
Climate & Reject & Confirmed \\
\hline
\end{tabular}

excluded from the analyses due to violations of the experiment instructions support this argument. In all these cases, a few alternatives were vetoed and then an agreement reached or the negotiation terminated without agreement, rather than all alternatives vetoed and the decision to agree or break-off the negotiation only made based on the last remaining possible solution as required by the experiment instructions in the veto treatment. ${ }^{1}$

The above discussed results need to be considered in the light of the limitations of the conducted study. First of all, only one specific negotiation case with two levels of complexity and one specific variant out of many possible for the offer exchange and veto procedure were considered in this exploratory study. Clearly the validity of the results would benefit from replications with other cases, other negotiation problems and other variants of negotiation procedures for offer exchange and veto. For example, the effects of offer and veto procedures that address a multiple issue negotiation issue by issue instead of packages offers, that cover options for all issues, could be analyzed and compared to the results of this initial study. Other forms of complexity besides the number of options in the issues could be considered, e.g. the number of issues themselves or if the negotiation is complicated based on how integrative or distributive the negotiation problem is. Furthermore, the negotiation by veto procedure described in this paper is not applicable to negotiation problems consisting of at least one continuous issue, such as time or money, which causes an infinite number of possible alternatives. Discretization of the options in an actually continuous issue could nevertheless enable the usage of negotiation by veto in such negotiation problems. Consider for example delivery time, an issue also used in the experiments in this study, which is, as it is based on time, an inherently continuous issue. However, while negotiators might have different preferences for a delivery time of one or two weeks, or even one or two days, they might be indifferent between delivery times varying only in the area of hours or minutes-though this might vary depending on the topical domain of the negotiation problem. Discretization in such cases can lead to a finite number of options in the issue and possible solutions in the negotiation problem without loss of information. Finally, the laboratory studies were conducted with student participants. Although external validity and generalizability of results would clearly benefit from replications of the experiments with practitioners or expert negotiators, literature suggests that negotiation novices and experts

\footnotetext{
${ }^{1}$ We thank the two anonymous reviewers for pointing to this explanation and for further valuable feedback.
} 
show very similar decision patterns Neale and Northcraft (1986) and no significant differences in performance Herbst and Schwarz (2011) (Table 10).

Given these results future research has to investigate how negotiation by veto could be improved to increase applicability and acceptance in negotiations and realize it is positive influence on negotiation outcomes. The experiments showed that many observations had to be excluded as the dyads were not able to follow or deliberately not followed the veto procedure. Those who did, reached fewer agreements compared to the offer exchange procedure. Besides the violations of the veto procedure and the terminated negotiations in this treatment, especially the low selfreported satisfaction values for all satisfaction measures evaluated indicate that the procedure in its current form is pre-mature. Furthermore, the process which leads to the outcomes must not be neglected. Future research has to investigate if and to what extent participants deviated from rational behavior in the veto procedure, i.e. why and how often negotiators not eliminated the worst of the remaining alternatives, and what effect these deviations had on agreement prospects and quality.

The veto procedure showed to be especially beneficial to negotiation outcomes if the problem is complex, i.e. features a large number of possible alternatives. However, in this case the manual elimination of solution alternatives might be a laborious and time consuming procedure. Exchanging offers allows for faster progress by larger concession steps at the risk of potentially missing possibilities for joint gains. The veto procedure on the other hand takes time but does not bear this risk as all potential solutions are systematically considered before they are consecutively eliminated until only one remains as a potential agreement. This makes the veto procedure a candidate strategy for automated negotiation Jennings et al. (2001), where the negotiation is delegated to software agents and proceeds very fast so time or computational capabilities are of minor importance. Time-based, e.g. Faratin et al. (1998), and problem-based, e.g. Filzmoser (2010a), Filzmoser (2010b), concession strategies-amongst others-already found their use in automated negotiation research. With the SNT even another improvement-based progression negotiation approach is already implemented for automated negotiation of specific complex contracts Klein et al. (2003). The veto procedure might be a viable alternative for automated negotiation. Future research should implement it and compare its performance with that of concession-based negotiation strategies in simulations and computational experiments in addition to the laboratory experiments with human participants presented in this paper. Preliminary results of a simulation study with time-based concession, problem-based concession and vetoing strategies applied to the negotiation problems introduced in Mumpower (1991) indicate that the performance of strategies, measured by negotiation time and negotiation outcome, varies significantly for some negotiation problems Filzmoser (2018).

Acknowledgements Open access funding provided by TU Wien (TUW).

Open Access This article is distributed under the terms of the Creative Commons Attribution 4.0 International License (http://creativecommons.org/licenses/by/4.0/), which permits unrestricted use, distribution, and reproduction in any medium, provided you give appropriate credit to the original author(s) and the source, provide a link to the Creative Commons license, and indicate if changes were made. 


\section{References}

Abonyi G (1983) Filtering: an approach to generating the information base for collective choice. Manag Sci 29(4):409-418

Bartos OJ (1977) Simple model of negotiation. J Confl Resolut 21(4):565-579

Bazerman MH, Russ LE, Yakura E (1987) Post-settlement settlements in two-party negotiations. Negot J 3:283-292

Curhan JR, Elfenbein HA, Xu H (2006) What do people value when they negotiate? Mapping the domain of subjective value in negotiation. J Personal Soc Psychol 91(3):493-512

Dupont C, Faure GO (2002) The negotiation process. In: Kremenyuk VA (ed) International negotiation: analysis, approaches, issues. Jossey-Bass, San Francisco, pp 39-63

Faratin P, Sierra C, Jennings NR (1998) Negotiation decision functions for autonomous agents. Robot Auton Syst 24:159-182

Filzmoser M, Vetschera R (2008) A classification of bargaining steps and their impact on negotiation outcomes. Group Decis Negot 17:421-443

Filzmoser M (2010) Simulation of automated negotiation. Springer, Vienna

Filzmoser M (2010) Automated vs. human negotiation. Int J Artif Intell 4(10):64-77

Filzmoser M (2018) Systematic computational comparison of automated negotiation protocols and strategies, In: Jiang X, Xu H, He S, Ke GY (eds) Proceedings of the 18th International Conference on Group Decision and Negotiation, Nanjing University of Aeronautics and Astronautics, 9.6.1813.6.18, Nanjing, China, pp 674-679

Fisher R (1978) International mediation: a working guide. International Peace Academy, New York

Fisher R, Ury W (1981) Getting to yes. Houghton-Mifflin, Boston

Gettinger JR, Filzmoser M, Koeszegi ST (2016) Why can't we settle again? Analysis of factors that influence agreement prospects in the post-settlement phase. J Bus Econ 86(4):413-440

Herbst U, Schwarz S (2011) How valid is negotiation research based on student sample groups? New insights into a long-standing controversy. Negot J 27(2):147-170

Jennings NR, Faratin P, Lomuscio AR, Parsons S, Wooldridge M, Sierra C (2001) Automated negotiation: prospects, methods and challenges. Group Decis Negot 10(2):199-215

Kelley HH (1966) A classroom study of the dilemmas in interpersonal negotiations. In: Archibald K (ed) Strategic interaction and conflict: original papers and discussion. Institute of International Studies, Berkeley, pp 49-73

Kersten GE, Noronha SJ (1999) WWW-based negotiation support: design, implementation, and use. Decis Support Syst 25:135-154

Klein M, Faratin P, Syayama H, Bar-Yam Y (2003) Negotiating complex contracts. Group Decis Negot 12(2):111-125

Lewicki RJ, Litterer JA, Saunders DM (1994) Negotiation, 2nd edn. Irwin, Burr Ridge

Moulin H (1979) Dominance solvable voting schemes. Econometrica 47(6):1337-1351

Müller D (1978) Voting by veto. J Public Econ 10(1):57-75

Mumpower M (1991) The judgement policies of negotiators and the structure of negotiation problems. Manag Sci 37(10):1304-1324

Neale MA, Northcraft GB (1986) Experts, amateurs, and refrigerators: comparing expert and amateur negotiators in a novel task. Organ Behav Hum Decis Process 38(3):305-317

Pruitt D (1981) Negotiation behavior. Academic Press, New York

Raiffa H (1985) Post-settlement settlement. Negot J 1:9-12

Rangaswamy A, Shell GR (1997) Using computers to realize joint gains in negotiations: toward an electronic bargaining table. Manag Sci 43(8):1147-1163

Teich JE, Wallenius H, Wallenius J (1994) Advances in negotiation science. Trans Oper Res 6(1):55-94

Tripp TM, Sondak H (1992) An evaluation of dependent variables in experimental negotiation studies: impasse rates and Pareto efficiency. Organ Behav Hum Decis Process 51:273-295

Tutzauer F (1992) The communication of offers in dyadic bargaining. In: Putnam L, Roloff ME (eds) Communication and negotiation. Sage, Newbury Park, pp 67-82

Yuval F (2002) Sophisticated voting under the sequential voting by veto. Theory Decis 53:343-369

Zartman IW (1977) Negotiation as a joint decision-making process. J Confl Resol 21(4):619-638 